\title{
Differential proteomics of the synovial membrane between bilateral and unilateral knee osteoarthritis in surgery-induced rabbit models
}

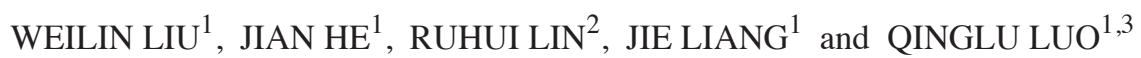 \\ ${ }^{1}$ Department of Physiotherapy, College of Rehabilitation Medicine; ${ }^{2}$ Medical Experimental Center, \\ Fujian Academy of Integrative Medicine, Fujian University of Traditional Chinese Medicine, \\ Fuzhou, Fujian 350122; ${ }^{3}$ Department of Orthopaedic Rehabiliation, Affiliated Rehabilitation Hospital \\ Fujian University of Traditional Chinese Medicine, Fuzhou, Fujian 350003, P.R. China
}

Received July 16, 2015; Accepted June 21, 2016

DOI: $10.3892 / \mathrm{mmr} .2016 .5511$

\begin{abstract}
The present study investigated the differential proteomics of synovial membranes between bilateral and unilateral anterior cruciate ligament transection (ACLT) in rabbits with knee osteoarthritis (KOA), in order to elucidate the pathological biomarkers of different degrees of KOA. A total of 6 New Zealand rabbits were randomly divided into groups A and B (three rabbits per group). The two groups were subjected to bilateral and unilateral ACLT, respectively. A total of 6 weeks following surgery, proteins were extracted from the knee joint synovial membranes of KOA rabbits and were separated by two-dimensional polyacrylamide gel electrophoresis. The differentially expressed proteins in the OA synovial membranes were selected for further analysis by linear ion trap-Fourier transform ion cyclotron resonance mass spectrometry. Ten protein spots were identified to be different between the synovial membranes of the bilateral and unilateral KOA rabbits. Protein disulfide-isomerase and creatine kinase M-type were identified in the unilateral KOA rabbit synovial membranes. Serum albumin (three spots), lumican, $\alpha$-2-HS-glycoprotein and three uncharacterized proteins were identified in the synovial membranes of the bilateral KOA rabbits. The differential proteomic expression demonstrated the different biomarkers associated with bilateral and unilateral KOA, and indicated that spontaneous and secondary KOA require diverse methods of treatment; thus the underlying mechanism of KOA requires further investigation.
\end{abstract}

Correspondence to: Dr Qinglu Luo, Department of Physiotherapy, College of Rehabilitation Medicine, Fujian University of Traditional Chinese Medicine, 1 Qiu Yang Road, Fuzhou, Fujian 350122, P.R. China

E-mail: luo_qinglu@126.com

Key words: differential proteomics, synovial membrane, osteoarthritis

\section{Introduction}

Osteoarthritis (OA) is one of the most common causes of musculoskeletal disability. It is characterized by progressive degeneration of articular cartilage and synovitis is a significant contributor, which contributes to the development of OA (1). Diagnosis of OA using magnetic resonance imaging indicates synovial hyperplasia proximal to cartilage lesions, particularly in the bursa suprapatellaris and posterior cruciate ligament of the knee (2). A previous study proposed that the synovitis is a significant cause of pain and oedema in OA patients (3). Although interleukin-6 (IL-6), cluster of differentiation 4 (CD4), CD8+ T-cells and adipocytokines (such as adiponectin and leptin) are vital inflammatory factors for the process of synovitis $(4,5)$, the molecular mechanisms of the progressive degeneration of articular synovial membrane in OA remain to be fully elucidated. Previously, alterations in the transcriptomes of OA synovial membranes were investigated using DNA microarray or reverse transcription-quantitative polymerase chain reaction analysis $(6,7)$. However, alterations in mRNA expression levels have been reported to not always correlate well with the protein levels due to post-transcriptional regulation, post-translational modification and differential stability of proteins (8).

The use of proteomics, during which entire proteins in tissues or cells are identified and quantified directly, has been identified as a valuable method for elucidating the molecular basis of disease etiology. Recently, the proteome of human articular chondrocytes, synovial fluid, serum or urine was characterized by two-dimensional polyacrylamide gel electrophoresis (2-DE) and tandem mass spectrometry of cultured chondrocytes isolated from normal cartilage (9-13). However, to the best of our knowledge, few proteomic studies regarding the articular synovial membrane have been conducted (14), and the present study have improved knowledge of the proteome of the synovial membrane, and provided a foundation for further investigation of the pathology of synovial membrane diseases.

Studies regarding the molecular and cellular mechanisms of OA have evaluated bilateral or unilateral joint tissue samples without considering the distinction between spontaneous and 
secondary OA (10,15-18). However, the majority of spontaneous (resulting from the aging process) and secondary (traumatic) knee OA (KOA) cases occur in bi/unilateral knee joints, respectively (19), and the pathological process and treatment for the disease may differ between spontaneous and secondary KOA $(20,21)$. Furthermore, certain studies proposed that proteomics may be important in the treatment of OA $(9,22)$. Thus, the present study hypothesizes that the mechanisms of proteomic alterations in the progressive destruction of articular synovial membrane in spontaneous and secondary KOA are different. The profile of proteins selectively extracted from rabbit synovial membrane samples of bi/unilateral KOA were compared by two-dimensional gel electrophoresis (2-DE) and mass spectral analysis to highlight requirements for establishing diverse treatments for OA resulting from the aging process and traumatic $\mathrm{OA}$.

\section{Materials and methods}

Animals. New Zealand White (NZW) rabbits were supplied by the Fujian University of Traditional Chinese Medicine (Fuzhou, China) animal testing center [batch no. SCXK (Shanghai) 2012-0011]. The NZW rabbits, 3 male and 3 female (age, 6 months; weight, 2.5-3.0 kg), were provided with a standard laboratory diet with drinking water and housed in individual cages under a 12 -h light/dark cycle at $20-26^{\circ} \mathrm{C}$. The present study complied with national legislation and with the Ministry of Health of the People's Republic of China Guide for the Care and Use of Laboratory Animals (23). Local ethical committee approval was obtained for the current study from the ethics committee of the Fujian University of Traditional Chinese Medicine. The NZW rabbits were sacrificed by air embolism 6 weeks following the surgery for OA model induction.

Animal grouping. All animals were randomly divided into groups A and B, with 3 rabbits per group, and SPSS 13.0 statistical software was used (SPSS, Inc., Chicago, IL, USA).

KOA model. The A and $\mathrm{B}$ group rabbits were subjected to bilateral and unilateral anterior cruciate ligament transection (ACLT), respectively. Briefly, the rabbits were administered with intraperitoneal injections of $5 \%$ chloral hydrate ( $3 \mathrm{ml} / \mathrm{kg}$; Qingdao Yulong Algae Co., Ltd., Qingdao, China). in order to sedate and anesthetize them appropriately. The right knee was shaved, sterilized, draped under sterile conditions and a medial arthrotomy was performed. The patella was then dislocated, and the ACL was isolated and transected. ACLT was confirmed by the surgeon and an observer using the Lachman test. Following irrigation using sterile saline solution, the wounds were closed in layers and treated with antiseptic. Rabbits were provided with the appropriate postoperative care and allowed to move freely in individual cages.

Specimen collection for synovial membrane proteomic detection. The rabbits were sacrificed 6 weeks subsequent to surgery, and the synovial membrane of the operative right knee joint was dissected in group A and B rabbits. The samples were maintained for subsequent evaluation in a nitrogen canister.
Protein extraction of the synovial membrane. Synovial membrane samples were ground into powdered tissue using a pestle and mortar, and transferred into a homogenizer. Lysates (500 $\mu \mathrm{l} / 100 \mathrm{mg}$ ) were added to the homogenate; RNase $(50 \mu \mathrm{g} / \mathrm{ml})$, DNase $(200 \mu \mathrm{g} / \mathrm{ml})$ and $10 \mu \mathrm{l} / 1 \mathrm{ml}$ lysate were added, and maintained at $4^{\circ} \mathrm{C}$ for $15 \mathrm{~min}$. The tissue was then centrifuged at $12,000 \mathrm{x} \mathrm{g}$ for $60 \mathrm{~min}$ at $4^{\circ} \mathrm{C}$. The supernatant was collected and the protein concentration was determined using the 2-D Quant kit (GE Healthcare Life Sciences, Uppsala, Sweden) according to the manufacturer's protocols. The tissues were refrigerated at $-70^{\circ} \mathrm{C}$.

\section{$2-D E$}

Protein solubility and dry strip swelling. Lysis buffer [500 $\mu \mathrm{l}$; $7 \mathrm{~mol} / \mathrm{l}$ urea, $2 \mathrm{~mol} / 1$ thiourea, 4\% CHAPS, $1 \%$ dithiothreitol (DTT), $0.2 \%$ NP-40, $1 \%$ ampholine (pH 4-6) and 1\% ampholine $(\mathrm{pH} 3.5-10)]$ was added into the protein solution extraction, which was then vibrated for $5 \mathrm{~h}$. Dissolution of the protein solution extraction was conducted using a $200 \mathrm{~W}$ ultrasonic instrument for $200 \mathrm{sec}$. The protein solution extraction was centrifuged at $20,000 \times \mathrm{g}$ for $20 \mathrm{~min}$ at $4^{\circ} \mathrm{C}$. The supernatant was collected and the protein concentration was measured using the Bradford Protein assay kit (Nanjing Jiancheng Bioengineering Institute, Nanjing, China). The rehydration solution [800 $\mu \mathrm{l} ; 8 \mathrm{~mol} / 1$ urea, 2\% CHAPS, 0.5\% ampholine ( $\mathrm{pH} 4-7$ ), $0.002 \%$ Bromophenol Blue and $800 \mu \mathrm{g}$ protein solution) was added into the protein electrophoresis tank and the dry strip was immersed ( $\mathrm{pH} 4-7 ; 18 \mathrm{~cm}$ ), gum down and incubated room temperature overnight.

Isoelectric focusing electrophoresis. The expanded $12 \%$ gel was placed into the gel strip slot of the isoelectric focusing electrophoresis apparatus and the gel was covered with covering oil. The electrophoresis parameters were as follows: $500 \mathrm{~V}$ for $2 \mathrm{~h}$ (gradient); 1,000 V for $1 \mathrm{~h}$ (gradient); 8,000 V for $2.5 \mathrm{~h}$ (step).

Gel strip equilibration. The gel strip was equilibrated twice following isoelectric focusing electrophoresis, for $15 \mathrm{~min}$ each time, with gentle agitation. The liquid components for the initial equilibration were as follows: $50 \mathrm{mmol} / 1 \mathrm{Tris}-\mathrm{Hcl}$ (pH 8.8), 6 mol/1 urea, 30\% glycerol, 2\% sodium dodecyl sulfate (SDS) and 1\% DTT. The second equilibrium liquid components were as follows: $50 \mathrm{mmol} / 1$ Tris- $\mathrm{Hcl}(\mathrm{pH} \mathrm{8.8)}$, $6 \mathrm{~mol} / \mathrm{l}$ urea, $30 \%$ glycerol, $2 \%$ SDS and $2.5 \%$ idoacetamide.

SDS-polyacrylimide gel electrophoresis. The balance gel was layered on the top of the spacer gel, taking care to avoid trapped bubbles, and the gel strip was fixed with $0.5 \%$ agarose. The electrode buffer was added following solidification of the agarose, and electrophoresis was performed until the Bromophenol Blue indicator reached the bottom of the separation gel. The concentrations of separation gel and spacer gel were 15 and $7 \%$ respectively, and the current was $30 \mathrm{~mA}$.

Staining. Coomassie Brilliant Blue R-250 was used for staining. The gel was solidified for $1 \mathrm{~h}$ in fixation fluid comprised of $50 \%$ anhydrous ethanol and $10 \%$ glacial acetic acid. The fixation fluid was removed and the gel was stained with $0.1 \%$ Coomassie Brilliant Blue R-250 and vibrated for $10 \mathrm{~h}$. The 
stain was removed by rinsing the gel twice with distilled water and adding destainer (30\% anhydrous ethanol and $8 \%$ glacial acetic acid) and vibrating. The destainer was replaced until the background of the gel was clear.

Silver staining and image scanning. Following silver staining and coloration the ImageScanner (GE Healthcare Life Sciences) was used to obtain the 2-DE images. The protein spots were counted using ImageMaster 2D Platinum software, version 3.0 (GE Healthcare Life Sciences). Automatic identification of protein spots was conducted with the software, however, if the boundary between protein spots were clear, they were segmented into two spots.

Gel image comparison. The distribution of protein isoelectric points of the two types of protein extraction were compared according to the 2-DE gel image. Bandscan 5.0 (Glyko, Inc., Novato, CA, USA) was used as comparison software.

In-gel digestion. Samples were spotted onto a MALDI target plate with an equal volume of matrix solution, containing $5 \mathrm{mg} / \mathrm{ml} \alpha$-cyano-4-hydroxycinnamic acid in $50 \%$ acetonitrile and $0.1 \%$ trifluoroacetic acid. An AutoFlex speed MALDI TOF/TOF MS (Bruker Corporation, Billerica, MA, USA) was used with a mass accuracy of 50 ppm following external calibration. The samples were analyzed in MS mode (for generation of peptide mass fingerprints) as well as in TOF/TOF mode (for fragmentation analysis of the highest intensity peaks). MS spectra were transformed into peak lists using the software flexAnalysis version 3.0 (Bruker Corporation). The peak lists of the MS and MS/MS spectra were merged using BioTools version 3.0 software (Bruker Corporation).

Protein detection. The amino acid sequence tags obtained from each peptide fragmentation in MS/MS analyses were used to calculate match scores and search for protein candidates in three groups using Mascot software, version 2.3.01 from Matrix Science (http://www.matrixscience.com). The retrieval parameters were as follows: Type of search, MS/MS Ion Search; enzyme, trypsin; fixed modification, Carbamidomethyl (C); variable modification: Gln->pyro-Glu (N-term Q), Oxidation (M); mass values, monoisotopic; protein mass, unrestricted; peptide mass tolerance, $\pm 0.1 \mathrm{Da}$; and fragment mass tolerance, $\pm 0.1 \mathrm{Da}$.

\section{Results}

2-DE imaging. Samples from the two groups underwent 2-DE three times $(500 \mu \mathrm{g} / \mathrm{sample})$ in the same environment, the images were scanned and the three images were identified to be comparable in each group (Figs. 1 and 2). The match scores were $82.1 \pm 1 \%$ in group $\mathrm{A}$ and $83.2 \pm 2 \%$ in group B.

Differential analysis of protein spots. A total of 10 different protein spots were identified by 2-DE of KOA synovial membrane samples in groups A and B; the homologous proteins, putative molecular weight and isoelectric point, and protein scores were determined (Fig. 3). Out of the 10 proteins, certain protein spots were identified to be the same, such as NO3, NO7 and NO8 (serum albumin). In samples of unilateral KOA synovial membrane, NO1 was protein disulfide-isomerase and NO2 was creatine kinase (CK) M-type. NO6 was identified as lumican, NO10 was $\alpha$-2-HS-glycoprotein (AHSG) and NO4, NO5, and NO9 were designated as uncharacterized proteins from the bilateral KOA synovial membrane samples (Table I).

\section{Discussion}

The synovial membrane is located within joint spaces and aids in the maintenance of normal joint function. Synovial membranes produce and secrete hyaluronan to lubricate the tissues of the joint, and serve an important role in the nutrition of cartilage, in addition to absorbing inflammation factors. Synovial fibrosis is a major contributor to joint stiffness in OA, which is elevated in OA and is key in the onset and persistence of synovial fibrosis. The process of synovial membrane lesions (from early inflammation to synovial hyperplasia), and the generation of inflammatory mediators and cytokines results in cartilage damage. Therefore, it is hypothesized that investigating and treating the cartilage alone in $\mathrm{OA}$ is not sufficient. Further investigation is required regarding the prevention of $\mathrm{OA}$, to include the consideration of diverse pathogenic factors and taking an interdisciplinary approach, with the synovial membrane becoming a novel treatment target for OA, which may prevent joint structure damage and improve the clinical symptoms. In recent years, in order to further clarify the diagnostic biomarkers and prognostic indicators in different diseases, increasing numbers of studies are referring to the use of proteomics. Proteomes of degenerative/inflamed synovial membranes from rheumatoid arthritis (RA) and OA and a chronic arthritic condition, spondyloarthropathy were previously investigated using 2-DE followed by tandem mass spectrometry (15).

To date, there are few studies regarding the proteomics of the synovial membrane. Furthermore, to the best of our knowledge, there are no studies reporting the differences between proteomics of the synovial membrane in spontaneous and secondary OA induced by a bilateral and unilateral ACLT model of KOA. Thus, the present study aimed to elucidate the differences in the proteomics of the synovial membrane using 2-DE in spontaneous and secondary KOA rabbit models, in order to establish the diverse remedies for OA resulting from the aging process and traumatic $\mathrm{OA}$ in the future. The results illustrated that the proteomics of the synovial membrane in the spontaneous and secondary KOA models were different. The proteins, disulfide-isomerase and CK M-type, were identified in the unilateral ACLT synovial membrane tissue, and serum albumin (three protein spots), lumican and AHSG were observed in the bilateral ACLT synovial membrane tissue. In addition, three proteins spots were uncharacterized in the bilateral ACLT synovial membrane.

Protein disulfide-isomerases (PDIs) have been reported in different tumors and 19 family members have been identified. The function of PDI is to catalyze oxidative folding of novel peptide chains in the endoplasmic reticulum, in addition to participating in calcium homeostasis and antigen presentation. Procollagen and thyroglobulin, which are associated with PDI have been identified in previous studies (24). Li et al (25) investigated mechanical-stress loading-induced OA of the articulatio mandibularis, and identified PDI in the mandibular 

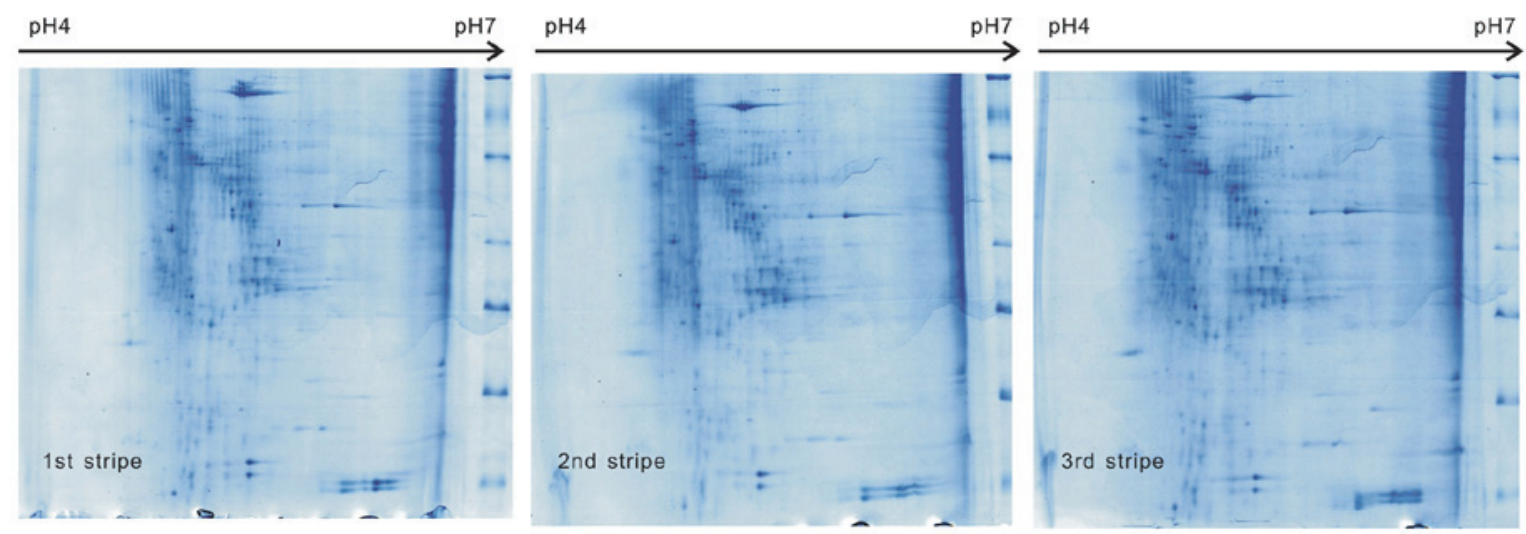

3 stripe of bilateral proteomics

Figure 1. Images of three stripes from 2-DE of group A following bilaterial anterior cruciate ligament transection. The image results for the three stripes from the same 2-DE are consistent, demonstrating that 2-DE of the synovium from group A was successful. 2-DE, two-dimensional polyacrylamide gel electrophoresis.
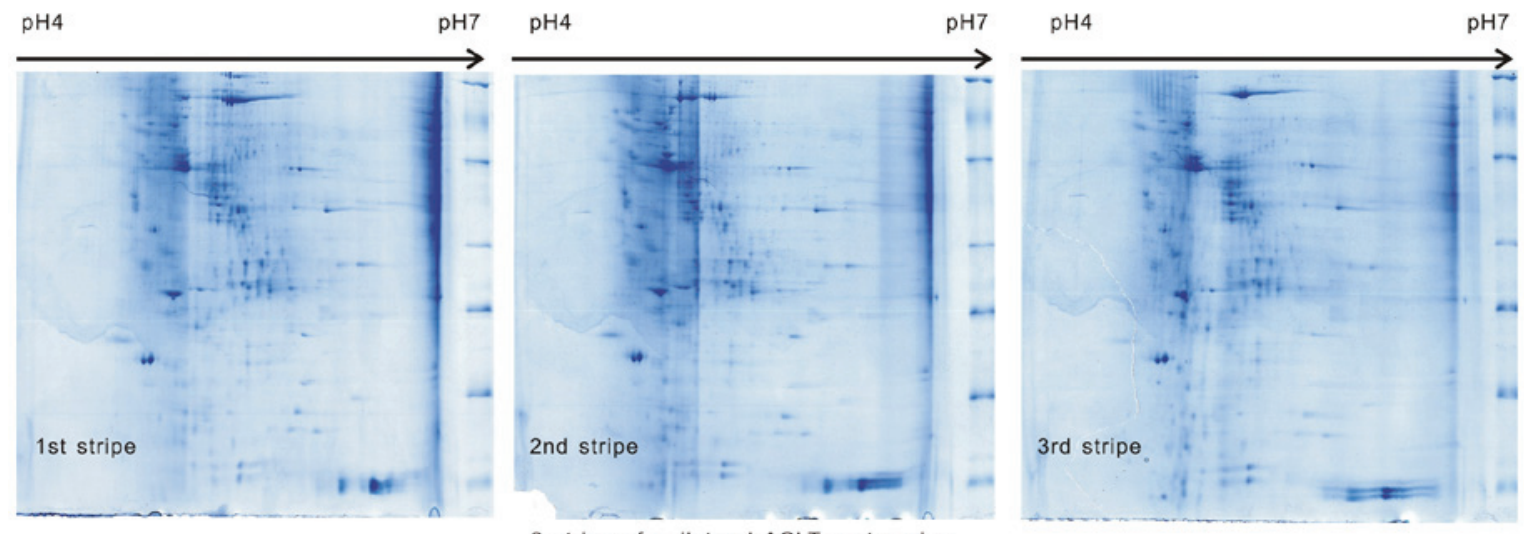

3 stripe of unilateral ACLT proteomics

Figure 2. Images of three stripes from 2-DE of group B following unilaterial anterior cruciate ligament transection. The image results for the three stripes from the same 2-DE are consistent, demonstrating that 2-DE of the synovium from group B was successful. 2-DE, two-dimensional polyacrylamide gel electrophoresis. ACLT, anterior cruciate ligament transection.

cartilage of rats. However, whether the synovial membrane of rabbits with KOA contains PDI has not, to the best of our knowledge, been documented thus far. In the current study, PDI was identified in the synovial membrane of unilateral ACLT, while it was not identified in bilateral ACLT. PDI affects protein metabolism, calcium homeostasis and procollagen synthesis, which all impact upon the pathological alterations in the tissues of the KOA joint. Thus, it was hypothesized that the PDI reduces collagen synthesis, which accelerates the process of KOA due to increased load in the bilateral ACLT joint.

In addition, the CK M-type protein was identified in the synovial membrane of unilateral ACLT, while it was not identified in bilateral ACLT. The components of synovial fluid, which are secreted by the synovial membrane, can be exchanged, and enter the circulatory system via synovial membrane capillaries, are potential biomarkers that can be detected in blood and urine (26). Therefore, the present study proposed that the varied expression levels of the CK M-type protein in the synovial membrane results from the difference in severity of the synovial membrane lesion between bilateral and unilateral KOA. As a result of the difference in CK expression levels between bilateral and unilateral KOA, the density of $\mathrm{CK}$ in the serum is also varied; i.e. the $\mathrm{CK}$ density is downregulated in bilateral KOA. In addition, Chen et al (27) reported that the CK contents increase then reduce from onset to the later stages of RA. Eimre et al (28) proposed that OA was associated with increased sensitivity of mitochondrial respiration to ADP, causing a reduction in total activities of $\mathrm{CK}$ with marked reductions in the mitochondrial $\mathrm{CK}$ fraction. The authors suggested that due to degenerative remodeling occurring during the development of OA, these complexes become structurally and functionally impaired, resulting in increased access of exogenous ADP to mitochondria and dysfunction of the CK-phosphotransfer system. Borges et al (29) identified increased plasma activities of total CK (2.0-fold) in ballet dancers immediately after class, a finding that is significant in preventing the development of chronic conditions that are commonly observed in dancers, such as those with arthritis and synovitis.

Serum albumin is an essential material in cellular physical activity. Alterations in serum albumin content result in pathological alterations. Huang et al (30) demonstrated that the serum albumin status may be important in the utilization and metabolic turnover of plasma pyridoxal 5-phosphate in the presence of chronic inflammation and autoimmune disease, such 
Table I. A total of 10 representative proteins of knee osteoarthritis rabbits from groups A and B identified by mass spectrometry of two-dimensional polyacrylamide electrophoresis gels of the synovium.

\begin{tabular}{|c|c|c|c|c|}
\hline $\begin{array}{l}\text { Spot no. } \\
\text { (SSP) }\end{array}$ & $\begin{array}{l}\text { Accession no. } \\
\text { (in IPI_rabbit) }\end{array}$ & $\begin{array}{l}\text { Homologous } \\
\text { protein }\end{array}$ & $\begin{array}{l}\text { Putative Mr } \\
\text { (Da) / pI }\end{array}$ & $\begin{array}{c}\text { Protein } \\
\text { score }\end{array}$ \\
\hline \multicolumn{5}{|l|}{ Group A } \\
\hline NO1 & P21195 & Protein disulfide-isomerase & $57172 / 0.29$ & 207 \\
\hline $\mathrm{NO} 2$ & P00563 & Creatine kinase M-type & $43313 / 0.27$ & 1441 \\
\hline NO3 & G1U9S2 & Serum albumin & $70916 / 1.04$ & 546 \\
\hline NO4 & G1ST52 & Uncharacterized protein & $182182 / 0.25$ & 497 \\
\hline NO5 & G1SWS9 & Uncharacterized protein & $53679 / 2.13$ & 511 \\
\hline NO6 & G1SP97 & Lumican & $38736 / 0.59$ & 151 \\
\hline NO7 & G1U9S2 & Serum albumin & $70916 / 4.96$ & 1657 \\
\hline NO8 & G1U9S2 & Serum albumin & $70916 / 1.50$ & 545 \\
\hline \multicolumn{5}{|l|}{ Group B } \\
\hline NO9 & G1SP97 & Uncharacterized protein & $50151 / 3.51$ & 728 \\
\hline NO10 & G1SGQ5 & $\alpha$-2-HS-glycoprotein & $39539 / 2.25$ & 358 \\
\hline
\end{tabular}

Mr, molecular weight; pI, isoelectric points; SSP, secondary structure of protein.

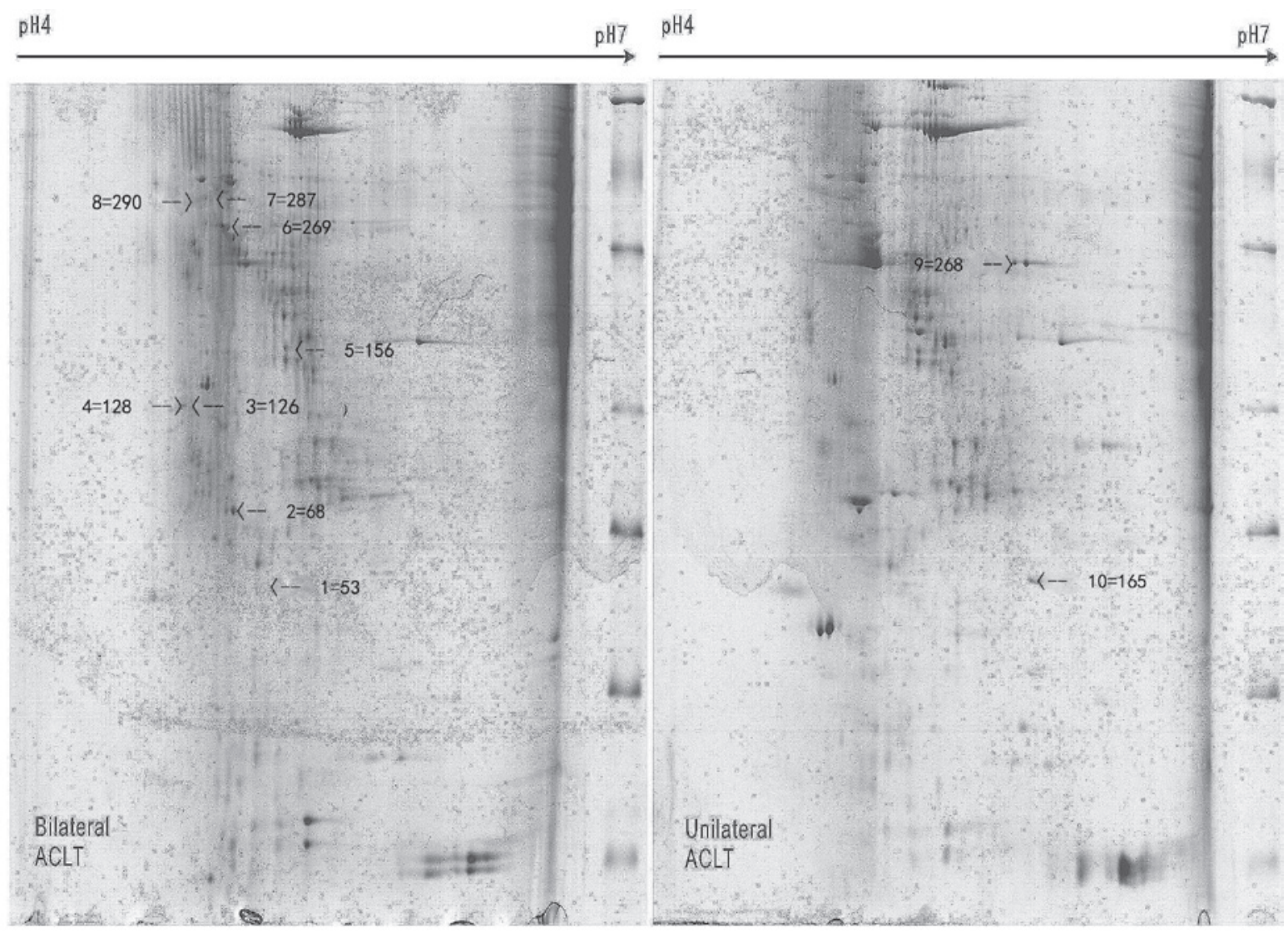

Figure 3. Protein spot distribution in synovial membranes from groups A (bilateral ACLT) and B (unilateral ACLT). ACLT, anterior cruciate ligament transection. Values are molecular weight divided by isoelectric point.

as in patients with RA. However, there are few investigations regarding the association between $\mathrm{OA}$ and serum albumin (31). In the present study, the serum albumin level in the synovial membrane of bilateral ACLT was observed (it was not identified in unilateral ACLT) with three of the protein spots identified as serum albumin; thus, it was inferred that serum albumin levels may increase in the early-middle stage (6-week ACLT model) in order to increase the elimination of inflammatory factors. Therefore, regulating serum albumin levels may present as a novel method for treating OA. 
Lumican is a leucine-rich proteoglycan and component of the extracellular matrix. Lumican and fibromodulin regulate the assembly of collagens into higher order fibrils in connective tissues. Jepsen et al (32) hypothesized that lumican and fibromodulin were candidate genes and key in the pathogenesis of certain types of Ehlers-Danlos syndrome and other connective tissue disorders. Previously, numerous lumican studies were regarding tumors, with few studies focusing on KOA (31-34). However, the association between lumican and OA or RA has been reported; Seki et al (33) identified that cultured RA fibroblastoid synoviocytes contain lumican protein, which encodes extracellular matrix components. Further investigation of lumican and fibromodulin may facilitate with the treatment of RA. In the present results, two protein spots were identified as lumican in the synovial membrane of bilateral ACLT, however not in unilateral ACLT (34). Fernández-Puente et al (34) identified serum protein biomarkers for moderate and severe OA, and identified six proteins that were only modulated in moderate OA, 13 proteins that were only modulated in severe $\mathrm{OA}$ and 7 that were modulated in the two; one of which was lumican. The authors indicated that the specificity and selectivity of these candidate proteins required validation prior to the development of novel molecular diagnostic or prognostic tests for OA. Melrose et al (35) observed that the fragmentation of small leucine-rich proteoglycans was increased in the degenerate osteoarthritic articular cartilage and menisci when compared with the articular cartilage of a normal knee. The authors suggested that specific decorin and fibromodulin core protein fragments in degenerate meniscus and/or human articular cartilage may be of value as biomarkers of disease, and further research may identify them as therapeutic targets. Clements et al (36) identified that the expression levels of lumican genes were increased in OA cartilage. Therefore, the present study proposed that lumican would be upregulated in $\mathrm{OA}$, and that regulating lumican expression in the synovial membrane may present as a novel treatment method for OA, consistent with a previous study (35).

The role of AHSG in the augmentation of neutrophil phagocytosis by macrophages, thus acting as an anti-inflammatory molecule, was reported in 1961 (37). Heiss et al (38) suggested that AHSG is a systemic inhibitor of precipitation of basic calcium phosphate, preventing unwanted calcification, and that AHSG domain D1 is most efficient in inhibiting basic calcium phosphate precipitation (38). Liu et al (39) demonstrated that the AHSG gene may contribute to bone size variation at the hip in a Chinese population. In addition, Nishio et al (40) observed that AHSG exerted mild inhibitory effects on calcium oxalate crystallization, and that low urinary concentrations of prothrombin F1 and osteopontin may contribute to stone formation. According to further findings, numerous studies have identified that AHSG is a non-specific opsonin, with its serum level being demonstrated to vary in patients who have experienced trauma, or who have diabetes mellitus. Lebreton et al (41) identified that the serum level of AHSG was negatively correlated with the acute phase reactants. A previous study by Mbuyi-Muamba et al (42) reported that treatment of RA did not appear to modify AHSG plasma levels; thus, the probable biological role of AHSG in RA is debated. However, Saroha et al (43) reported that the level of plasma AHSG was reduced by two-fold in RA patients when compared with healthy control subjects. To date, the association between OA and AHSG has required further elucidation. In the present study, the synovial membrane sample contained AHSG in the bilateral ACLT group while it was not observed in the unilateral ACLT group. The present study proposes that during the onset of $\mathrm{OA}$, the synovial membrane expression of AHSG may be reduced for anti-inflammatory purposes or to induce the hyperostosis.

The proteomics in the synovial membrane of spontaneous and secondary KOA models were compared in the present study. As all 10 proteins have not been reported in the synovial membrane, their functions in KOA can only be hypothesized. The present study identified PDI and CK M-type in the unilateral KOA model, but not in the bilateral KOA model (and more severe pathological changes than unilateral KOA). PDI may accelerate the process of KOA, as it regulates protein metabolism, calcium homeostasis and procollagen synthesis. The levels of CK may reflect the different disease phases or the degree of pathology of KOA; with upregulated CK concentrations in the early phase/light KOA and downregulated $\mathrm{CK}$ concentrations at the mid to late phase/severe KOA.

Serum albumin, lumican, AHSG and three uncharacterized proteins were observed in the bilateral KOA model, but not in the unilateral KOA model. It is hypothesized that the serum albumin levels increase to inhibit the KOA process. The lumican levels in the synovial membrane may induce cell proliferation in connective tissues resulting in synovial hyperplasia, and AHSG proteins in the synovial membrane may be secreted into the synovial fluid, stimulating bone formation and resulting in hyperostosis.

In conclusion, the present results demonstrate the differential proteomic expression and indicate the diverse pathomechanisms between bilateral and unilateral KOA, highlighting that spontaneous and secondary KOA require diverse methods of treatment. Regulation of PDI and CK M-type expression levels may be necessary in secondary KOA or in the early phase of spontaneous KOA, and reduced lumican and AHSG levels in spontaneous KOA, particularly at the mid to late phase, as the majority of patients are at this stage upon OA diagnosis. However, further investigations regarding the different mechanisms of synovial membrane proteomics in spontaneous and secondary KOA are required.

\section{Acknowledgements}

The present study was financially supported by the National Natural Science Foundation of China (grant no. 81273774).

\section{References}

1. Krasnokutsky S, Attur M, Palmer G, Samuels J and Abramson SB: Current concepts in the pathogenesis of osteoarthritis. Osteoarthritis Cartilage 16 (Suppl 3): S1-S3, 2008.

2. Hayashi D, Roemer FW, Katur A, Felson DT, Yang SO, Alomran F and Guermazi A: Imaging of synovitis in osteoarthritis: Current status and outlook. Semin Arthritis Rheum 41: 116-130, 2011.

3. Scanzello CR and Goldring SR: The role of synovitis in osteoarthritis pathogenesis. Bone 51: 249-257, 2012.

4. Pawłowska J, Mikosik A, Soroczynska-Cybula M, Jóźwik A, Łuczkiewicz P, Mazurkiewicz S, Lorczyński A, Witkowski JM and Bryl E: Different distribution of CD4 and CD8 T cells in synovial membrane and peripheral blood of rheumatoid arthritis and osteoarthritis patients. Folia Histochem Cytobiol 47: 627-632, 2009. 
5. Presle N, Pottie P, Dumond H, Guillaume C, Lapicque F, Pallu S, Mainard D, Netter P and Terlain B: Differential distribution of adipokines between serum and synovial fluid in patients with osteoarthritis. Contribution of joint tissues to their articular production. Osteoarthritis Cartilage 14: 690-695, 2006.

6. Okabe T, Ohmori Y, Tanigami A, Hishigaki H, Suzuki Y, Sugano S, Kawaguchi A, Nakaya H and Wakitani S: Detection of gene expression in synovium of patients with osteoarthritis using a random sequencing method. Acta Orthop 78: 687-692, 2007.

7. Lambrecht S, Verbruggen G, Elewaut D and Deforce D: Differential expression of alpha B-crystallin and evidence of its role as a mediator of matrix gene expression in osteoarthritis. Arthritis Rheum 60: 179-188, 2009.

8. Anderson NL, Matheson AD and Steiner S: Proteomics: Applications in basic and applied biology. Curr Opin Biotechnol 11: 408-412, 2000

9. Ruiz-Romero C and Blanco FJ: Proteomics role in the search for improved diagnosis, prognosis and treatment of osteoarthritis. Osteoarthritis Cartilage 18: 500-509, 2010.

10. Guo D, Tan W, Wang F, Lv Z, Hu J, Lv T, Chen Q, Gu X, Wan B and Zhang Z: Proteomic analysis of human articular cartilage: Identification of differentially expressed proteins in knee osteoarthritis. Joint Bone Spine 75: 439-444, 2008.

11. Mobasheri A: Osteoarthritis year 2012 in review: Biomarkers. Osteoarthritis Cartilage 20: 1451-1464, 2012.

12. Fernández-Costa C, Calamia V, Fernández-Puente $\mathrm{P}$ Capelo-Martinez JL, Ruiz-Romero C and Blanco FJ: Sequential depletion of human serum for the search of osteoarthritis biomarkers. Proteome Sci 10: 55, 2012.

13. Henrotin Y, Gharbi M, Mazzucchelli G, Dubuc JE, De Pauw E and Deberg M: Fibulin 3 peptides Fib3-1 and Fib3-2 are potential biomarkers of osteoarthritis. Arthritis Rheum 64: 2260-2267, 2012.

14. Ruiz-Romero C, Calamia V, Carreira V, Mateos J, Fernández $\mathrm{P}$ and Blanco FJ: Strategies to optimize two-dimensional gel electrophoresis analysis of the human joint proteome. Talanta 80 $1552-1560,2010$

15. Hasegawa A, Nakahara H, Kinoshita M, Asahara H, Koziol J and Lotz MK: Cellular and extracellular matrix changes in anterior cruciate ligaments during human knee aging and osteoarthritis. Arthritis Res Ther 15: R29, 2013.

16. Revell PA, Mayston V, Lalor P and Mapp P: The synovial membrane in osteoarthritis: A histological study including the characterisation of the cellular infiltrate present in inflammatory osteoarthritis using monoclonal antibodies. Ann Rheum Dis 47 300-307, 1988

17. Willett TL, Kandel R, De Croos JN, Avery NC and Grynpas MD Enhanced levels of non-enzymatic glycation and pentosidine crosslinking in spontaneous osteoarthritis progression. Osteoarthritis Cartilage 20: 736-744, 2012.

18. Lambrecht S, Verbruggen G, Verdonk PC, Elewaut D and Deforce D: Differential proteome analysis of normal and osteoarthritic chondrocytes reveals distortion of vimentin network in osteoarthritis. Osteoarthritis Cartilage 16: 163-173, 2008

19. Johnson VL and Hunter DJ: The epidemiology of osteoarthritis Best Pract Res Clin Rheumatol 28: 5-15, 2014.

20. Millington SA, Li B, Tang J, Trattnig S, Crandall JR, Hurwitz SR and Acton ST: Quantitative and topographical evaluation of ankle articular cartilage using high resolution MRI. J Orthop Res 25: 143-151,2007.

21. Ratzlaff CR and Liang MH: New developments in osteoarthritis. Prevention of injury-related knee osteoarthritis: Opportunities for the primary and secondary prevention of knee osteoarthritis. Arthritis Res Ther 12: 215, 2010.

22. Mobasheri A: Applications of proteomics to osteoarthritis, a musculoskeletal disease characterized by aging. Front Physiol 2: 108,2011

23. The Ministry of Science and Technology of the People's Republic of China: Guidance suggestion of caring laboratory animals. Beijing, P.R. China, 2006.

24. Vandenbroeck K, Martens E and Alidza I: Multichaperone complexes regulate the folding of interferon-gamma in the endoplasmic reticulum. Cytokine 33: 264-273, 2006.

25. Li H, Zhang XY, Wu TJ, Cheng W, Liu X, Jiang TT, Wen J, Li J, Ma QL and Hua ZC: Endoplasmic reticulum stress regulates rat mandibular cartilage thinning under compressive mechanical stress. J Biol Chem 288: 18172-18183, 2013.
26. Williams A, Smith JR, Allaway D, Harris P, Liddell S and Mobasheri A: Applications of proteomics in cartilage biology and osteoarthritis research. Front Biosci (Landmark Ed) 16 : 2622-2644, 2011

27. Chen X and Wang F: Variation and clinical significance of serum cardiac enzymes in patients with rheumatoid arthritis. China Medical Herald 10: 51-52, 2013.

28. Eimre M, Puhke R, Alev K, Seppet E, Sikkut A, Peet N, Kadaja L, Lenzner A, Haviko T, Seene T, et al: Altered mitochondrial apparent affinity for ADP and impaired function of mitochondrial creatine kinase in gluteus medius of patients with hip osteoarthritis. Am J Physiol Regul Integr Comp Physiol 290: R1271-R1275, 2006

29. Borges LS, Bortolon JR, Santos VC, de Moura NR, Dermargos A, Cury-Boaventura MF, Gorjão R, Pithon-Curi TC and Hatanaka E: Chronic inflammation and neutrophil activation as possible causes of joint diseases in ballet dancers. Mediators Inflamm 2014: 846021, 2014

30. Huang SC, Wei JC, Lin PT, Wu DJ and Huang YC: Plasma pyridoxal 5-phosphate is not associated with inflammatory and immune responses after adjusting for serum albumin in patients with rheumatoid arthritis: A preliminary study. Ann Nutr Metab 60: 83-89, 2012.

31. Izai M, Miyazaki S, Murai R, Morioka Y, Hayashi H, Nishiura M and Miura K: Prorenin-renin axis in synovial fluid in patients with rheumatoid arthritis and osteoarthritis. Endocrinol Jpn 39: 259-267, 1992

32. Jepsen KJ, Wu F, Peragallo JH, Paul J, Roberts L, Ezura Y, Oldberg A, Birk DE and Chakravarti S: A syndrome of joint laxity and impaired tendon integrity in lumican and fibromodulin deficient mice. J Biol Chem 277: 35532-35540, 2002.

33. Seki T, Selby J, Häupl T and Winchester R: Use of differential subtraction method to identify genes that characterize the phenotype of cultured rheumatoid arthritis synoviocytes. Arthritis Rheum 41: 1356-1364, 1998

34. Fernández-Puente P, Mateos J, Fernández-Costa C, Oreiro N, Fernández-López C, Ruiz-Romero C and Blanco FJ: Identification of a panel of novel serum osteoarthritis biomarkers. J Proteome Res 10: 5095-5101, 2011.

35. Melrose J, Fuller ES, Roughley PJ, Smith MM, Kerr B Hughes CE, Caterson B and Little CB: Fragmentation of decorin, biglycan, lumican and keratocan is elevated in degenerate human meniscus, knee and hip articular cartilages compared with age matched macroscopically normal and control tissues. Arthritis Res Ther 10: R79, 2008.

36. Clements DN, Fitzpatrick N, Carter SD and Day PJ: Cartilage gene expression correlates with radiographic severity of canine elbow osteoarthritis. Vet J 179: 211-218, 2009.

37. Schmid K and Burgi W: Preparation and properties of the human plasma Ba-alpha2-glycoproteins. Biochim Biophys Acta 47: 440-453, 1961.

38. Heiss A, DuChesne A, Denecke B, Grötzinger J, Yamamoto K, Renné T and Jahnen-Dechent W: Structural basis of calcification inhibition by alpha 2-HS glycoprotein/Fetuin-A Formation of colloidal calciprotein particles. J Biol Chem 278: 13333-13341, 2003.

39. Liu YJ, Liu XH, Lei SF, Li MX and Deng HW: Alpha2-HS glycoprotein gene is associated with bone size at the hip in Chinese. Yi Chuan Xue Bao 32: 1128-1135, 2005

40. Nishio S, Hatanaka M, Takeda H, Iseda T, Iwata $H$ and Yokoyama M: Analysis of urinary concentrations of calcium phosphate crystal-associated proteins: Alpha2-HS-glycoprotein, prothrombin F1, and osteopontin. J Am Soc Nephrol 10 (Suppl 14): S394-S396, 1999

41. Lebreton JP, Joisel F, Raoult JP, Lannuzel B, Rogez JP and Humbert G: Serum concentration of human alpha 2 HS glycoprotein during the inflammatory process: Evidence that alpha $2 \mathrm{HS}$ glycoprotein is a negative acute-phase reactant. J Clin Invest 64: 1118-1129, 1979.

42. Mbuyi-Muamba JM, Dequeker J and Stevens E: Alpha 2 HSglycoprotein in rheumatoid arthritis. Its plasma concentration and possible biological role. Rev Rhum Mal Osteoartic 49: 515-518, 1982 (In French).

43. Saroha A, Kumar S, Chatterjee BP and Das HR: Jacalin bound plasma O-glycoproteome and reduced sialylation of alpha 2-HS glycoprotein (A2HSG) in rheumatoid arthritis patients. PLoS One 7: e46374, 2012. 